\title{
Photon decay in UHE air showers: a stringent bound on Lorentz violation
}

\author{
Fabian Duenkel, ${ }^{a, *}$ Marcus Niechciol $^{a}$ and Markus Risse ${ }^{a}$ \\ ${ }^{a}$ University of Siegen, Walter-Flex-Str. 3, Siegen, Germany \\ E-mail: duenkel@hep.physik.uni-siegen. de
}

\begin{abstract}
In extensive air showers induced by ultra-high energy (UHE) cosmic rays, secondary photons are expected to be produced at energies far above those accessible by other means. It has been shown that the decay of such photons, as possible in certain theories allowing the violation of Lorentz invariance, can lead to significant changes of the shower development. Based on observations of the average depth of shower maximum $\left\langle X_{\max }\right\rangle$, a stringent bound on the violation of Lorentz invariance has been placed in a previous work. In this contribution we include the shower-toshower fluctuations $\sigma\left(X_{\max }\right)$ as an additional observable. The combined comparison of $\left\langle X_{\max }\right\rangle$ and $\sigma\left(X_{\max }\right)$ to shower observations allows a much stricter test of the possible decay of UHE photons, improving the existing bound by a factor 50 .
\end{abstract}

$37^{\text {th }}$ International Cosmic Ray Conference (ICRC 2021)

July 12th - 23rd, 2021

Online - Berlin, Germany

\footnotetext{
${ }^{*}$ Presenter
} 


\section{Introduction}

It is known that the Standard model of Elementary Particle Physics (SM) does not explain everything that is observed in the universe. In many approaches to establish a more fundamental theory, deviations from exact Lorentz symmetry is possible (see e.g. [1]). The extremely high energies of cosmic rays and gamma rays have been used to test possible effects of Lorentz violation (LV), and some of the best limits on LV were obtained (see e.g.: [2-5]).

In this work we focus on isotropic, nonbirefringent LV in the photon sector in the case of a photon velocity larger than the maximum attainable velocity of standard Dirac fermions [6] which allows photon decay as a new process. Specifically, the impact of this type of LV on extensive air showers initiated by cosmic rays in the Earth's atmosphere is exploited, focussing on ultra-high energies (UHE) above $1 \mathrm{EeV}=10^{18} \mathrm{eV}$. This approach was first studied in [7] with an analytical ansatz, modifying the well-known Heitler model for electromagnetic cascades to include LV through photon decay. A significant impact on the longitudinal shower development of electromagnetic cascades was found. To study the impact of LV on air showers initiated by primary hadrons a full Monte Carlo (MC) ansatz was used in [2]. The modified decay of neutral pions due to LV [10] has also to be taken into account in the case of primary hadrons. Through comparison of the average atmospheric depth of the shower maximum $\left\langle X_{\max }\right\rangle$ for air showers with $\mathrm{LV}$ to shower observations a significant limit on LV has been determined. A significant improvement of this limit may be expected by adding further observables, which has already been noted in [2]. We extend that previous work by taking into account the shower-to-shower fluctuations $\sigma\left(X_{\max }\right)$ as an additional observable, which indeed leads to stricter constraints.

The theory background of LV in the context of this study and some relevant aspects of the previous analyses are briefly summarized in Sec. 2. The current analysis is presented in Sec. 3, in particular the methodology to compare simulations and data in more than one obervable and the result after application. Sec. 4 contains a discussion and a brief summary.

\section{Theory}

Lorentz violation (LV) in the photon sector can be achieved through a relatively simple extension of standard quantum electrodynamics (QED) by adding a single term which breaks Lorentz invariance but preserves CPT and gauge invariance to the Lagrange density [6]:

$$
\begin{aligned}
\mathcal{L}= & \underbrace{-\frac{1}{4} F^{\mu \nu} F_{\mu \nu}+\bar{\psi}\left[\gamma^{\mu}\left(i \partial_{\mu}-e A_{\mu}\right)-m\right] \psi}_{\text {standard QED }} \\
& \underbrace{-\frac{1}{4}\left(k_{F}\right)_{\mu \nu \rho \sigma} F^{\mu v} F^{\rho \sigma}}_{\text {CPT-even LV term }} .
\end{aligned}
$$

Natural units $(\hbar=c=1)$ and the Minkowski metric $g_{\mu \nu}(x)=\eta_{\mu \nu}=[\operatorname{diag}(+1,-1,-1,-1)]_{\mu \nu}$ are used in this entire work. The added tensor $\left(k_{F}\right)_{\mu \nu \rho \sigma}$ consists of 20 independent components, for the case of isotropic, nonbirefringent $\mathrm{LV}$ in the photon sector it is controlled by a single dimensionless 
parameter $\kappa$ in the following way:

$$
\left(k_{F}\right)_{\mu \lambda v}^{\lambda}=\frac{\kappa}{2}[\operatorname{diag}(3,1,1,1)]_{\mu \nu} .
$$

This changes the photon phase velocity to

$$
v_{\mathrm{ph}}=\frac{\omega}{|\vec{k}|}=\sqrt{\frac{1-\kappa}{1+\kappa}} c .
$$

Here, $c$ corresponds to the maximum velocity of a massive Dirac fermion, for negative values of $k$ the photon is faster than $c$, for positive values it is slower. In the case of $\kappa \neq 0$, processes which are forbidden in the SM become allowed. In this work we focus on the case of $\kappa<0$. Photons then become unstable above the energy threshold

$$
E_{\gamma}^{\mathrm{th}}(\kappa)=2 m_{e} \sqrt{\frac{1-\kappa}{-2 \kappa}} \simeq \frac{2 m_{e}}{\sqrt{-2 \kappa}} .
$$

The photons decay into electron-positron pairs, $m_{e} \simeq 511 \mathrm{keV}$ is the rest mass of the electron. The photon decay length drops to scales of centimeters and below right above this threshold, which corresponds to a quasi-instantaneous decay of photons into eletron-positron pairs [4, 8]. Since photons above threshold from astrophysical sources could not reach Earth, terrestial observations of gamma rays with energies of the order $100 \mathrm{TeV}$ from distant sources were used to impose an initial limit of $[4,8]$

$$
\kappa>-9 \times 10^{-16}(98 \% \mathrm{CL}) .
$$

Observations of higher-energy photons would be needed to improve this limit, but extensive searches for astrophysical (primary) photons with $\mathrm{PeV}$ or $\mathrm{EeV}$ energies resulted in no unambiguous photon detection at the corresponding energies(see e.g. [9]). However, photons with energies $\gg 100 \mathrm{TeV}$ are expected to be produced as secondary particles in air showers caused by the interaction of UHE hadrons in the Earth's atmosphere, especially in the start-up phase of the shower. Here, a modification of the particle behaviour due to LV can modify the development of the shower in a significant way[2, 7].

To be consistent with the LV-theory, the modification of the decay of neutral pions into two photons has to be considered. The decay time of neutral pions significantly increases in comparison to the SM for energies above $E_{\pi^{0}}^{\text {th }} \simeq 132 E_{\gamma}^{\text {th }}$, eventually becoming effectively stable (for more details, see [10]). Although the impact of this effect on the longitudinal shower development is minor, it is also included in the simulations used in this work.

Implementing these modifications in simulations of air showers, a strong dependence of the depth of the shower maximum $\left\langle X_{\max }\right\rangle$ on $\kappa$, as displayed in Fig. 1, was found [2] . Through comparison to data, the limit

$$
\kappa>-3 \times 10^{-19}(98 \% \mathrm{CL}) .
$$

could be placed. This improved the previous bound (5) from primary photon showers by a factor 3000 and proved the sensitivity of the new approach of testing secondary photons in primary hadron showers.

Still, an important limitation of bound (6) is related to the uncertain composition of the primary cosmic rays. Due to this, most conservatively a pure proton composition had to be assumed. This 

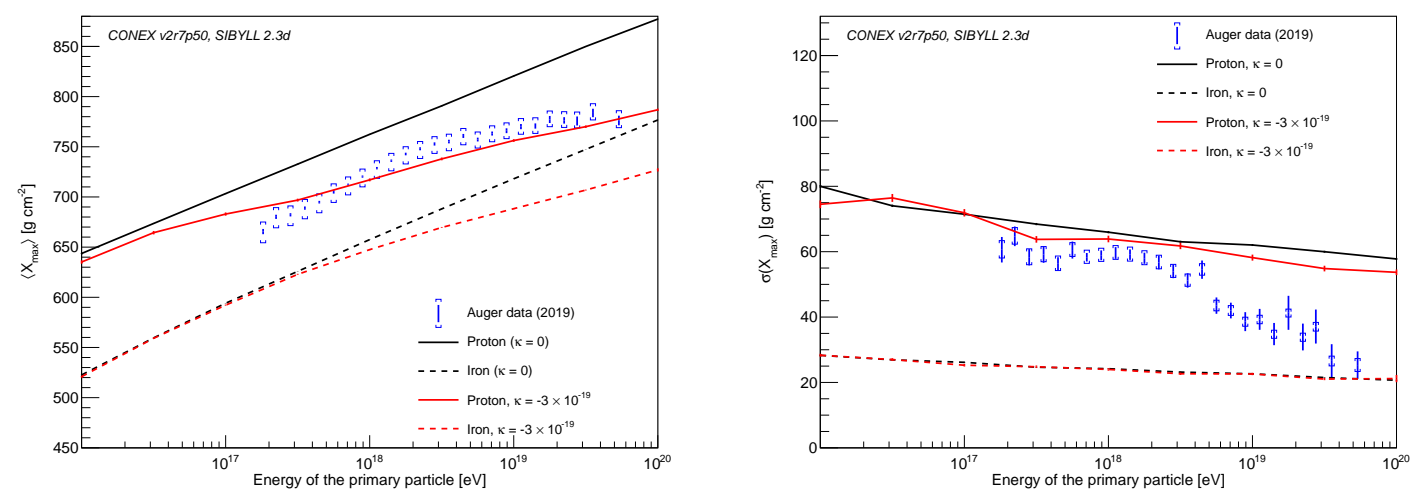

Figure 1: $\left\langle X_{\max }\right\rangle$ and $\sigma\left(X_{\max }\right)$ as a function of the primary energy for primary protons and iron nuclei for the absence of $\operatorname{LV}(\kappa=0)$ and for the previous best bound $\left(\kappa=-3 \times 10^{-19}\right)$. Shown are also measurements from the Pierre Auger Observatory [17], with both statistical uncertainties (shown as error bars) and systematic uncertainties (shown as boxes) included.

limitation could be overcome by including the shower fluctutations as an additional observable, as noted in [2]. In contrast to $\left\langle X_{\max }\right\rangle$, the fluctuations $\sigma\left(X_{\max }\right)$ show only a negligible dependence on $\kappa$ (see also Fig. 1). This may allow the exclusion of those composition assumptions that, for a given $\kappa$, might be able to reproduce either $\left\langle X_{\max }\right\rangle$ or $\sigma\left(X_{\max }\right)$ alone, but not both observables simultaneously.

\section{Analysis}

To analyze the impact of LV on the development of air showers, a full MC approach is used as was done in [2]. The MC code CONEX [11, 12] was modified to include the photon decay as well as the modified decay time of the neutral pion. Hadronic interactions are simulated with EPOS LHC [13] and QGSJET-II-04 [14] using CONEX v2r5p40 as well as with SIBYLL 2.3d [15] using CONEX v2r7p50. For all other settings, the default settings provided by the CONEX code are used. We checked that the values derived from simulations done with EPOS LHC and QGSJET-II-04 do not differ significantly between CONEX v2r5p40 and CONEX v2r7p50. The exact composition of cosmic ray particles is unknown, especially at high energies. To simulate any possible composition, four primary hadrons were chosen as representatives of their respective mass ranges. For this proton (mass number $A=1)$, helium $(A=4)$, oxygen $(A=16)$ and iron $(A=56)$ were chosen. The simulations done for these elements were combined at different proportions to simulate sets of shower data produced by different primary hadron compositions. The resulting $\left\langle X_{\max }\right\rangle$ value of any combination is the same as the weighted mean of the $\left\langle X_{\max }\right\rangle$ values of all components, while the $\sigma\left(X_{\max }\right)$ value of a set of showers with different primary hadrons is always greater than the weighted mean of the composites (see Fig. 2). This is due to the size of the shower-to-shower fluctuations increasing once showers induced by different particles with different mean shower depths are combined.

The possible values gained by this are then compared to measurements taken by the Pierre Auger Observatory [17]. To compare both observables at once, a 2D-confidence interval was used, the confidence level of $98 \%$ was chosen to assure comparabilty to the previously derived limits 


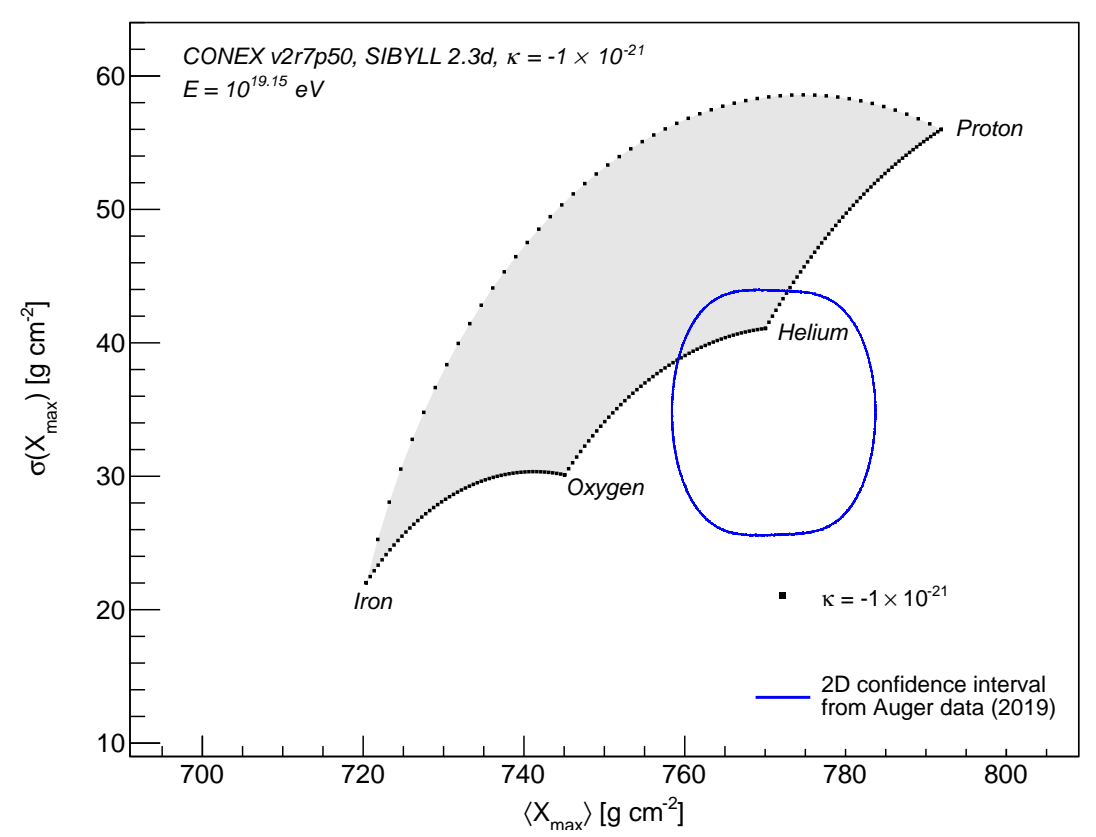

Figure 2: An outline of all possible $\left\langle X_{\max }\right\rangle / \sigma\left(X_{\max }\right)$ values for combinations of air showers induced by protons, helium, oxygen and iron for $\kappa=1 \times 10^{-20}$ and a primary particle energy of $10^{19.15} \mathrm{eV}$. Displayed are only proton-helium, helium-oxygen, oxygen-iron and iron-proton combinations, since all other possible combinations produce $\left\langle X_{\max }\right\rangle / \sigma\left(X_{\max }\right)$ values inside the umbrella-shaped area defined by those. Any point is differing $2 \%$ in composition from the neighboring points.

[2, 4]. For this the statistical and systematic uncertainties of the $\left\langle X_{\max }\right\rangle$ and $\sigma\left(X_{\max }\right)$ observations were approximated by Gaussian distributions (statistical) and equal distributions (systematic) and a contour line encompassing $98 \%$ of the distribution is drawn. The comparison is performed between all possible combinations of $\left\langle X_{\max }\right\rangle$ and $\sigma\left(X_{\max }\right)$ covered by the LV simulations and the Auger measurements. The illustration of such a comparison as well as the change of $\left\langle X_{\max }\right\rangle$ and $\sigma\left(X_{\max }\right)$ in dependence on $\kappa$ can be seen in Fig. 3.

An overlap between the areas representing the allowed values determined by the simulations and the Auger measurements shows that there are some primary hadron combinations fitting the Auger measurements. Reversely, if for a value of $\kappa$ an energy can be found for which there is no overlap, this $\kappa$ does not fit the measurements and can be excluded. This is repeated for the three models until a value $\kappa_{\text {crit }}$ is found where all $\kappa<\kappa_{\text {crit }}$ do not fit the measurements while all $\kappa>\kappa_{\text {crit }}$ do. The most conservative value is gained by using the SIBYLL 2.3d model, giving a new limit of $\kappa_{\text {crit }}=-6 \times 10^{-21}$ (at $98 \%$ C.L.) due to the combination of high $\left\langle X_{\max }\right\rangle$ values and low $\sigma\left(X_{\max }\right)$ values in the energy bins which determine $\kappa_{\text {crit }}$. A stricter limit is achieved using the EPOS model, resulting in a limit of $\kappa_{\text {crit }}=-5 \times 10^{-21}$. No limits on $\kappa$ can be derived with our method using QGSJETII, due to the much shallower showers simulated with this model.

This new bound of $\kappa_{\text {crit }}=-6 \times 10^{-21}$ is an improvement by a factor of 50 upon the previous bound [2]. 


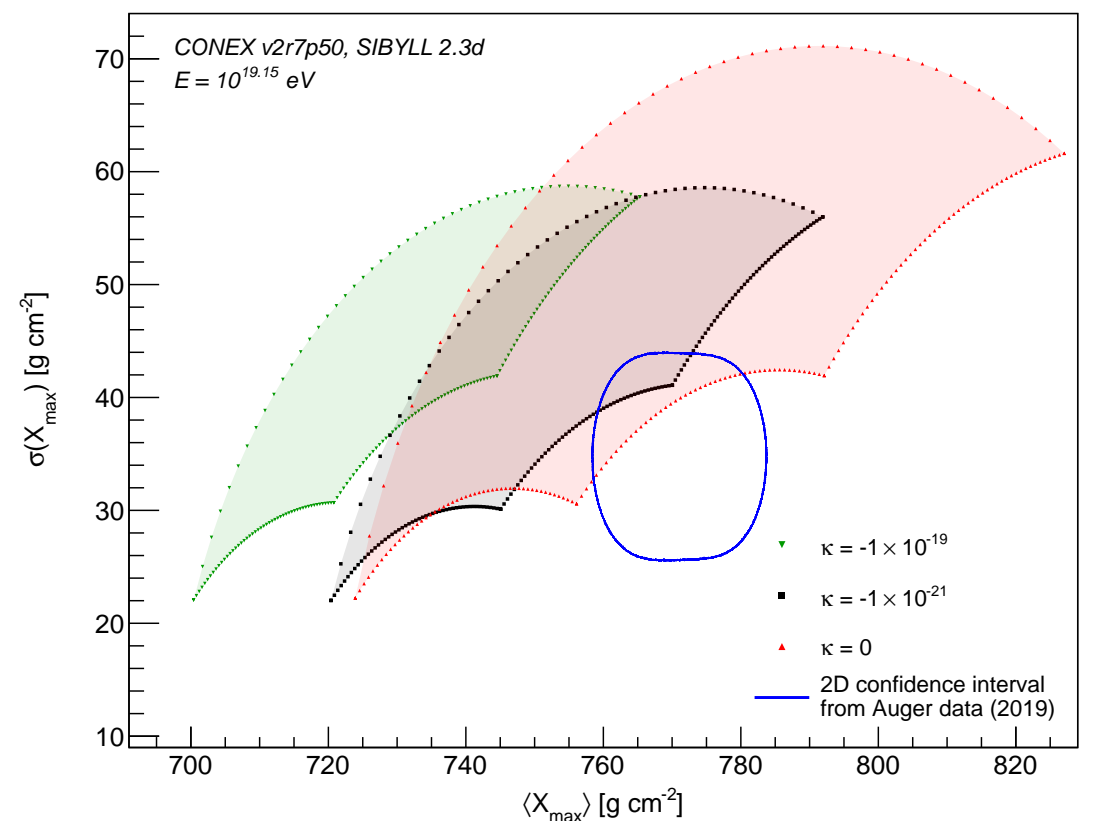

Figure 3: Comparison of $\left\langle X_{\max }\right\rangle$ and $\sigma\left(X_{\max }\right)$ derived by simulations which incorporate $\mathrm{LV}$ to the $2 \mathrm{D}$ confidence intervals given by the measurements of the Pierre Auger observatory at different values for $\kappa$ and a primary particle energy of $10^{19.15} \mathrm{eV}$.

\section{Discussion and outlook}

The important value for determining the bound obtained in this work is the Auger data in the energy range from $10^{19.1} \mathrm{eV}$ to $10^{19.2} \mathrm{eV}$ with a mean energy of $10^{19.15} \mathrm{eV}$. This is primarily due to the combination of the low $\sigma\left(X_{\max }\right)$ value at this energy paired with the comparatively small statistical uncertainty of $\sigma\left(X_{\max }\right)$. All simulated combinations compared to the confidence interval derived from Auger data for this critical combination of energy and $\kappa$ can be seen in Fig. 4. At this bound, the umbrella-shaped area which encompasses all possible combinations of $\left\langle X_{\max }\right\rangle$ and $\sigma\left(X_{\max }\right)$ allowed by the LV simulations almost "touches" the range allowed by the the Auger data. Since the value of $\left\langle X_{\max }\right\rangle$ increases with an increase of $\kappa$, this leads to a pure Helium compositon being the CR-composition which first matches the experimental data. Further improvements of the bound can be expected when the possible compositions of primary cosmic ray particles can be further restricted.

The improvement of the bound on $\kappa$ derived in this work in comparison to the previous bound [2] is not only due to the improvement of the method used but also due to the increase in data collected at the Auger Observatory and the corresponding decrease in statistical uncertainties between [16] and [17]. Using the method detailed in [2] would only yield minimal improvements in the bound on $\kappa$ due to the minimal changes in the energy range around $10^{18.4} \mathrm{eV}$. The main step forward here is the inclusion of $\sigma\left(X_{\max }\right)$ as a second observable.

The new bound is quite stable against the choice of the energy bin. A limit of $\kappa=-8 \times 10^{-21}$ would result from several other energy bins (in the range from $10^{18.8} \mathrm{eV}$ to $10^{19.1} \mathrm{eV}$ ). Formally, the energy bin at $10^{19.55} \mathrm{eV}$ - which we excluded here due to small statistics - would yield a somewhat 


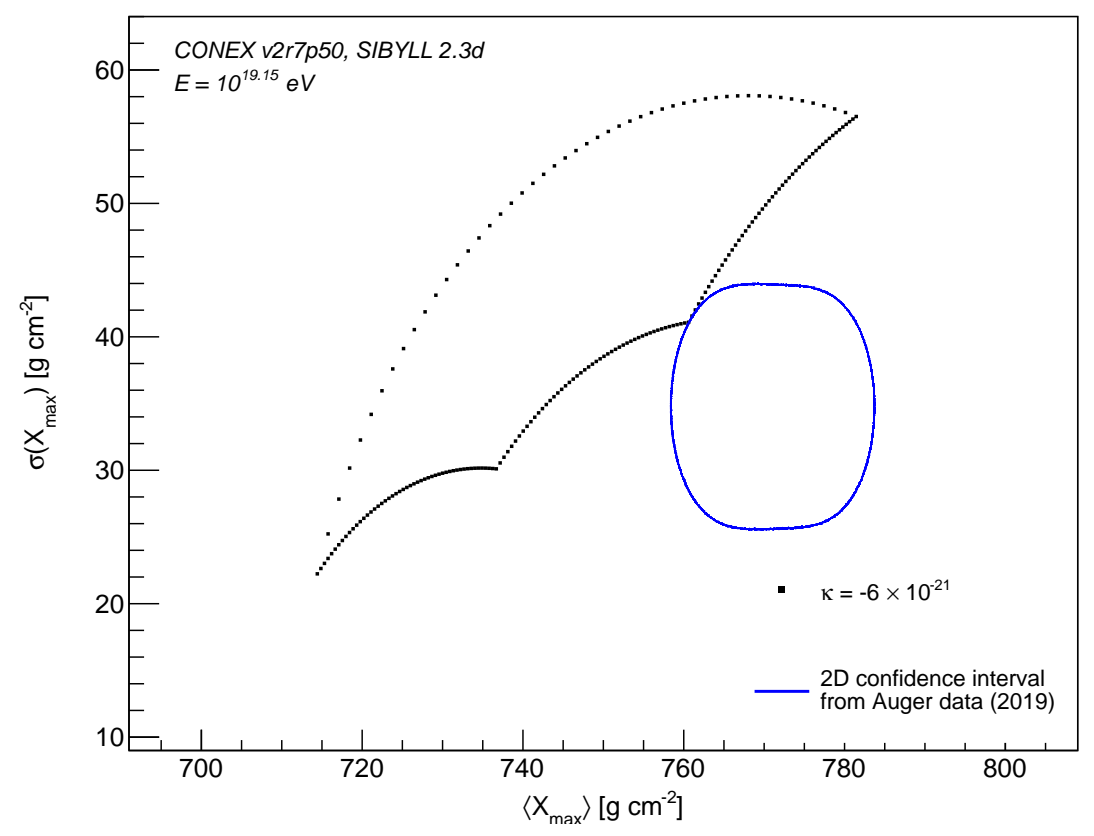

Figure 4: Comparison of $\left\langle X_{\max }\right\rangle$ and $\sigma\left(X_{\max }\right)$ derived by LV simulations to the $2 \mathrm{D}$ confidence interval given by the measurements of the Pierre Auger observatory for $\kappa=-6 \times 10^{-21}$ and a primary particle energy of $10^{19.15} \mathrm{eV}$.

stricter bound of $\kappa=-3 \times 10^{-21}$.

Further improvements upon this bound can come from additional data reducing experimental uncertainties. Improved bounds on $\kappa$ also appear possible if additional observables, such as $S(1000)$, are taken into account.

In summary, we tested the presence of the decay of secondary UHE photons that are expected to be produced in extensive air showers. Such decays, predicted as an LV effect in the theory framework considered, can affect the longitudinal shower development in a significant and well-defined way. Comparing to measurements by the Auger Observatory of $\left\langle X_{\max }\right\rangle$ and, as a further observable added in this work, the shower-to-shower fluctuation $\sigma\left(X_{\max }\right)$, a new bound on the LV-parameter $\kappa$ was derived. The new limit of $\kappa=-6 \times 10^{-21}$ (98\% C.L.) improves the previous bound by a factor 50 . Together with the present best limit on positive $\kappa[3,4]$, where the mere existence of UHE cosmic rays was exploited to exclude Vacuum Cherenkov radiation of the primary cosmic rays, $K$ is now bracketed by

$$
6 \times 10^{-20}>\kappa>-6 \times 10^{-21}(98 \% \mathrm{CL}) .
$$

In this work, we focused on the effect of $\kappa<0$ on the UHE shower development. The corresponding analysis of the effect of $\kappa>0$ will be reported on in a future study.

\section{Acknowledgments}

The many fruitful discussions with Frans R. Klinkhamer are greatly appreciated. We thank T. Pierog for his help in modifying the CONEX source code. This work was funded by the German Research Foundation (DFG project 408049454). 


\section{References}

[1] S. Liberati and L. Maccione, Ann. Rev. Nucl. Part. Sci. 59, 245 (2009).

[2] F. R. Klinkhamer, M. Niechciol, and M. Risse, Phys. Rev. D 96 (2017) 116011.

[3] F. R. Klinkhamer and M. Risse, Phys. Rev. D 77 (2008) 016002.

[4] F. R. Klinkhamer and M. Schreck, Phys. Rev. D 78 (2008) 085026.

[5] V. A. Kostelecký and N. Russell, Rev. Mod. Phys. 83 (2011) 11.

[6] V. A. Kostelecký and M. Mewes, Phys. Rev. D 66 (2002) 056005.

[7] J. S. Díaz, F. R. Klinkhamer and M. Risse, Phys. Rev. D 94 (2016) 085025.

[8] J. S. Díaz and F. R. Klinkhamer, Phys. Rev. D 92 (2015) 025007.

[9] M. Niechciol for the Pierre Auger Collaboration, PoS(ICRC2017) 517.

[10] F. R. Klinkhamer, arXiv:1610.03315 (2016).

[11] T. Bergmann, et al., Astropart. Phys. 26 (2007) 420.

[12] T. Pierog, et al., Nucl. Phys. Proc. Suppl. 151 (2006) 159.

[13] T. Pierog, et al., Phys. Rev. C 92 (2015) 034906.

[14] S. Ostapchenko, Phys. Rev. D 83 (2011) 014018.

[15] F. Riehn, et al., Phys. Rev. D 102 (2020) 063002.

[16] The Pierre Auger Collaboration, Phys. Rev. D 90 (2014) 122005.

[17] V. Verzi for the Pierre Auger Collaboration, PoS(ICRC2019) 450. 